\title{
mTOR signaling is activated by FLT3 kinase and promotes survival of FLT3-mutated acute myeloid leukemia cells
}

\author{
Weina Chen ${ }^{1}$, Elias Drakos ${ }^{1}$, loannis Grammatikakis ${ }^{1}$, Ellen J Schlette ${ }^{1}$, Jiang Li', Vasiliki Leventaki ${ }^{1}$, \\ Efi Staikou-Drakopoulou ${ }^{2}$, Efstratios Patsouris ${ }^{2}$, Panayiotis Panayiotidis ${ }^{3}$, L Jeffrey Medeiros ${ }^{1 *}$, George Z Rassidakis ${ }^{1,2^{*}}$
}

\begin{abstract}
Activating mutations of the FLT3 gene mediate leukemogenesis, at least in part, through activation of PI3K/AKT. The mammalian target of rapamycin (mTOR)-Raptor signaling pathway is known to act downstream of AKT. Here we show that the mTOR effectors, 4EBP1, p70S6K and rpS6, are highly activated in cultured and primary FLT3-mutated acute myeloid leukemia (AML) cells. Introduction of FLT3-ITD expressing constitutively activated FLT3 kinase further activates mTOR and its downstream effectors in BaF3 cells. We also found that mTOR signaling contributes to tumor cell survival, as demonstrated by pharmacologic inhibition of PI3K/AKT/mTOR, or total silencing of the $m T O R$ gene. Furthermore, inhibition of FLT3 kinase results in downregulation of mTOR signaling associated with decreased survival of FLT3-mutated AML cells. These findings suggest that mTOR signaling operates downstream of activated FLT3 kinase thus contributing to tumor cell survival, and may represent a promising therapeutic target for AML patients with mutated-FLT3.
\end{abstract}

\section{Background}

The FMS-like tyrosine kinase-3 (FLT3) receptor, also known as CD135, is a tyrosine kinase type III normally involved in hematopoietic progenitor cell proliferation, survival, and differentiation. Previous studies have shown that constitutive activation of FLT-3 is involved in leukemogenesis, partially through phosphorylation/ activation of the serine-threonine kinase AKT, which occurs in a substantial subset of acute myeloid leukemia (AML) cases [1,2]. A frequent mechanism of FLT3 activation is mutation of the FLT3 gene, either internal tandem duplication (ITD) within the juxtamembrane domain (FLT3-ITD), or point mutations within the activation loop of the second tyrosine kinase domain (FLT3-D835/836). FLT3 gene mutations are found in approximately one third of AML patients, and are associated with inferior prognosis [3].

The mammalian target of rapamycin (mTOR), an important downstream effector of AKT, is a master

\footnotetext{
* Correspondence: ljmedeiros@mdanderson.org; grasidak@med.uoa.gr 'Department of Hematopathology, The University of Texas M.D. Anderson Cancer Center, 1515 Holcombe Blvd., Houston, Texas 77030, USA Full list of author information is available at the end of the article
}

regulator of cell growth and metabolism [4]. There are two mTOR multi-protein complexes, mTOR-Raptor/ mTORC1 and mTOR-Rictor/mTORC2. mTOR-Raptor/ mTORC1 is sensitive to the natural macrolide rapamycin and regulates the rate of protein translation $[4,5]$. This regulation is accomplished, partially, through phosphorylation of the ribsosomal protein S6 kinase (p70S6K) and subsequent phosphorylation of the ribosomal protein $\mathrm{S} 6$ (rpS6), or phosphorylation and inactivation of the eukaryotic initiation factor $4 \mathrm{E}$ (eIF4E)-binding protein-1 (4E-BP1), dissociating 4E-BP1 from the RNA cap-binding protein eIF4E, thus promoting cap-dependent translation of mRNAs [4,5]. mTOR-Rictor/mTORC2, usually insensitive to rapamycin, phosphorylates AKT at serine residue 473, contributing to AKT activation, and establishing an autoregulatory loop [6].

Recent studies have shown that rapamycin, and its analogs, have substantial antitumor activity in hematologic malignancies, including AML $[7,8]$. However, the significance of FLT3 gene mutation in the activation of the mTOR pathway is not clear. In this study we hypothezised that the mTOR signaling pathway has an oncogenic role in FLT3-mutated AML cells. We show that 
mTOR signaling is highly activated in FLT3-mutated AML cell lines and primary cells. We also demonstrate that total inhibition of mTOR signaling results in cell death, specifically of FLT3-mutated AML cells, whereas inhibition of the FLT3 kinase results in downregulation of mTOR signaling. Our findings suggest that mTOR signaling operates downstream of mutated FLT3 kinase and that AML patients harboring FLT-3-mutations may benefit from experimental therapies that target mTOR signaling.

\section{Results and Discussion}

The mTOR signaling pathway is activated in FLT3mutated AML cells

Initially, we investigated the mTOR pathway activation status by immunohistochemistry and Western blot analysis of bone marrow samples and primary peripheral blood AML cells, respectively, in patients harboring FLT3 mutations. Imunohistochemical analysis showed that 10/12 (83\%) p-mTOR, 10/13 (77\%) p4E-BP1, 13/14 (93\%) p-p70S6K, and 11/12 (83\%) p-rpS6 indicating mTOR activation. All AML tumors $(\mathrm{n}=6)$ with dual mutations (FLT3-ITD and tyrosine kinase point mutations) were positive for $\mathrm{p}$-mTOR, p-p70S6K, and p-rpS6. Also, all AML tumors $(\mathrm{n}=15)$ expressed high levels of eIF4E (Figure 1A). This is the first immunohistochemistry study of mTOR signaling proteins in AML. We also showed activated mTOR signaling by Western blot analysis (Figure 1), in agreement with others [9]. To further investigate a possible causal association between FLT3 mutation and the mTOR activation status, we used stably transfected FLT3-mutant BaF3 cells. Introduction of FLT3-ITD in BaF3 cells resulted in activation of mTOR signaling (Figure 1).

\section{mTOR Signaling contributes to FLT3-mutated AML cell survival}

We assessed the role of mTOR signaling in the regulation of cell growth and survival of AML cell lines. Most previous studies investigating this issue were based on rapamycin treatment of AML cells resulting frequently in conflicting conclusions $[10,11]$. However, recently it was realized that rapamycin not only can indirectly and unpredictably inhibit the rapamycin-insensitive mTORC2 after prolonged treatment, but also frequently inhibits partially and not totally mTORC1, which is regarded as the specific target of rapamycin. For this reason, we employed the pharmacologic PI3K inhibitor LY294002, known to inhibit both the PI3K and MTOR kinase activity with the same kinetics, or total silencing of the $m T O R$ gene using an $m T O R$-specific siRNA [12,13].

As shown in the Figure 1, treatment with LY294002 resulted in a dose-dependent decrease in phosphorylation of AKT, mTOR, 4E-BP1, p70S6K, and rpS6, associated with reduced cell viability due to increased apoptosis (additional file 1, Figure S1). Although changes in the levels of mTOR signaling proteins are similar in both FLT3-mutated AML cell lines, it seems that MV4-11 cell viability depends more on mTOR signaling (Figure 1, Dright, and additional file 1, Figure S1) and this difference is also observed after combined inhibition of mTOR with rapamycin and FLT3 with GTP14564 (additional file 2, Figure S2). The biologic explanation for this finding is uncertain. The latter biologic effects were linked to downregulation of anti-apoptotic proteins involved in both extrinsic and intrinsic (mitochondrial) apoptotic pathways (additional file 1, Figure S1). In addition, transient transfection with $m T O R$-specific siRNA resulted in decreased phosphorylation/activation of 4EBP1, p70S6K, and rpS6 in a concentration-dependent manner (Figure 1). Of note, $m$ TOR silencing was associated with decreased levels of $\mathrm{p}-\mathrm{AKT}$ indicating that mTOR/Rictor complex may contribute to AKT activation in this cell system. Silencing of $m T O R$ also resulted in decreased viability of the AML cell lines, MV4-11 and MOLM13 (both mutated-FLT3) as opposed to Wt-FLT3 U937 cells which were unresponsive (Figure 1).

Taken together, our findings from mTOR gene silencing and pharmacologic studies suggest that mTOR signaling may contribute to the survival of AML cells harboring FLT3 mutations, and are concordant with the results of other studies $[8,14,15]$.

mTOR signaling acts downstream of mutated-FLT3 Kinase To further test this hypothesis, we evaluated the selective FLT3 inhibitor, GTP-14564, on mTOR signaling in AML cells. No changes in activation of mTOR signaling proteins, as well cell proliferation or death were observed in Wt-FLT3 U937 cells after treatment (Figure 2). However, treatment of the FLT3-mutated AML cell lines MV4-11 and MOLM13 resulted in a concentration-dependent decrease of p-mTOR, p-p70S6K, p-rpS6 and p-4EBP1. These changes were slightly more pronounced in the homozygously mutated MV4-11 compared with the heterozygously mutated MOML13 (Figure 2). Unexpectedly, AKT (p-AKT) activation was affected minimally or not at all (Figure 2). The biologic explanation for this finding is uncertain, however, the possibility that activation of the mTOR signaling pathway induced by mutated FLT3 kinase may partly involve AKT-independent mechanisms cannot be excluded. These changes were accompanied by a proportional decrease in cell proliferation, and increase in cell death (Figure 2). These biologic effects were further augmented when combined treatment with GTP14564 and Rapamycin was used (additional file 2, Figure S2). Downregulation of mTOR signaling accompanied by similar biologic effects were also observed after GTP14564 treatment of FLT3-mutated primary AML cells 

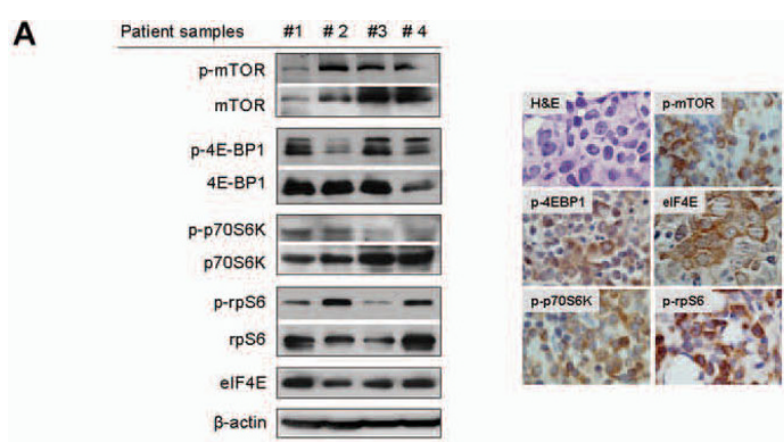

B

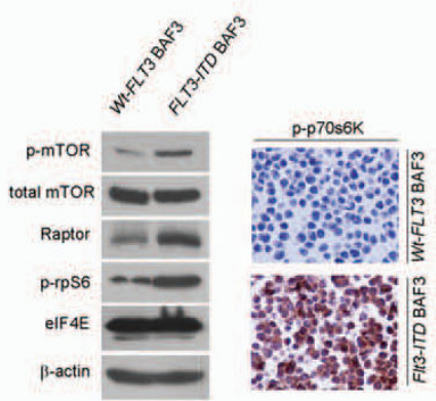

C

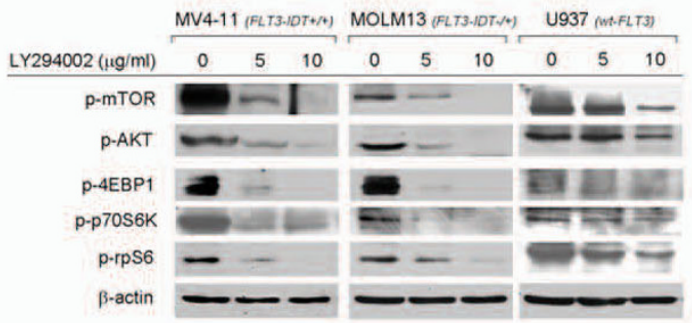

D
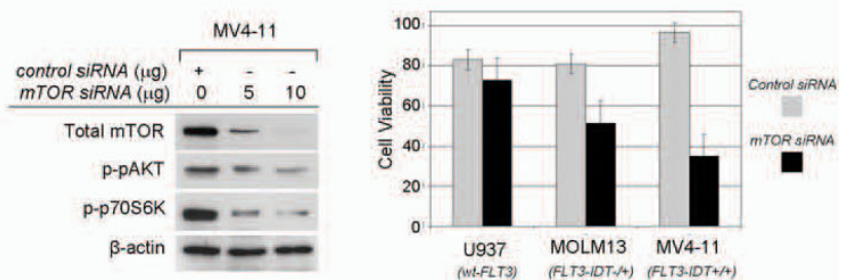

Figure 1 mTOR signaling pathway is activated and contributes to the survival of AML cells harboring mutated FLT-3. A. Western blot analysis of primary AML cells with mutated FLT3-ITD showed activation (phosphorylation) of mTOR kinase and downstream mTOR mediators (left panel). These data were confirmed also with immunohistochemistry on FLT3-mutated AML bone marrow samples (right panel). In positive cases, the majority of tumor cells show expression of phosphorylated (activated) mTOR, p70S6K, rpS6 and 4EBP1 with a cytoplasmic staining pattern (DAB chromogen, hematoxylin \& eosin [H\&E] counterstain, original magnification $\times 400$ ). B. A causal association between activation status of mTOR signaling pathway and activating mutations of FLT3 was further supported by the upregulation of mTOR, Raptor as well as phosphorylation (activation) of downstream effectors such as p70S6K and rpS6 proteins in BaF3 cells stably transfected with mutated FLT3 compared with BaF3 cells transfected with Wt-FLT3, as shown by Western blot analysis (left panel), or immunohistochemistry performed in cell blocks (right panel). C. Western blot analysis of AML cell lines showed that pharmacologic inhibition of PI3K and mTOR kinases by LY294002 resulted in downregulation of p-AKT and downstream mediators of the mTOR pathway. This effect was more pronounced in the MV4-11 and MOLM13 cell lines harboring mutated FLT3 comparing with the U937 cell line with Wt-FLT3. D. Silencing of the mTOR gene by transient transfection of mTOR-specific siRNA resulted in downregulation of mTOR and p-AKT signaling (left panel), associated with decreased survival of FLT3-mutated MOLM13 and MV4-11 cells by 30\% and 62\%, respectively, as compared with a small decrease by $13 \%$ of the Wt-FLT3 U937 cells $(p<0.05), 48$ hours after treatment (right panel). 


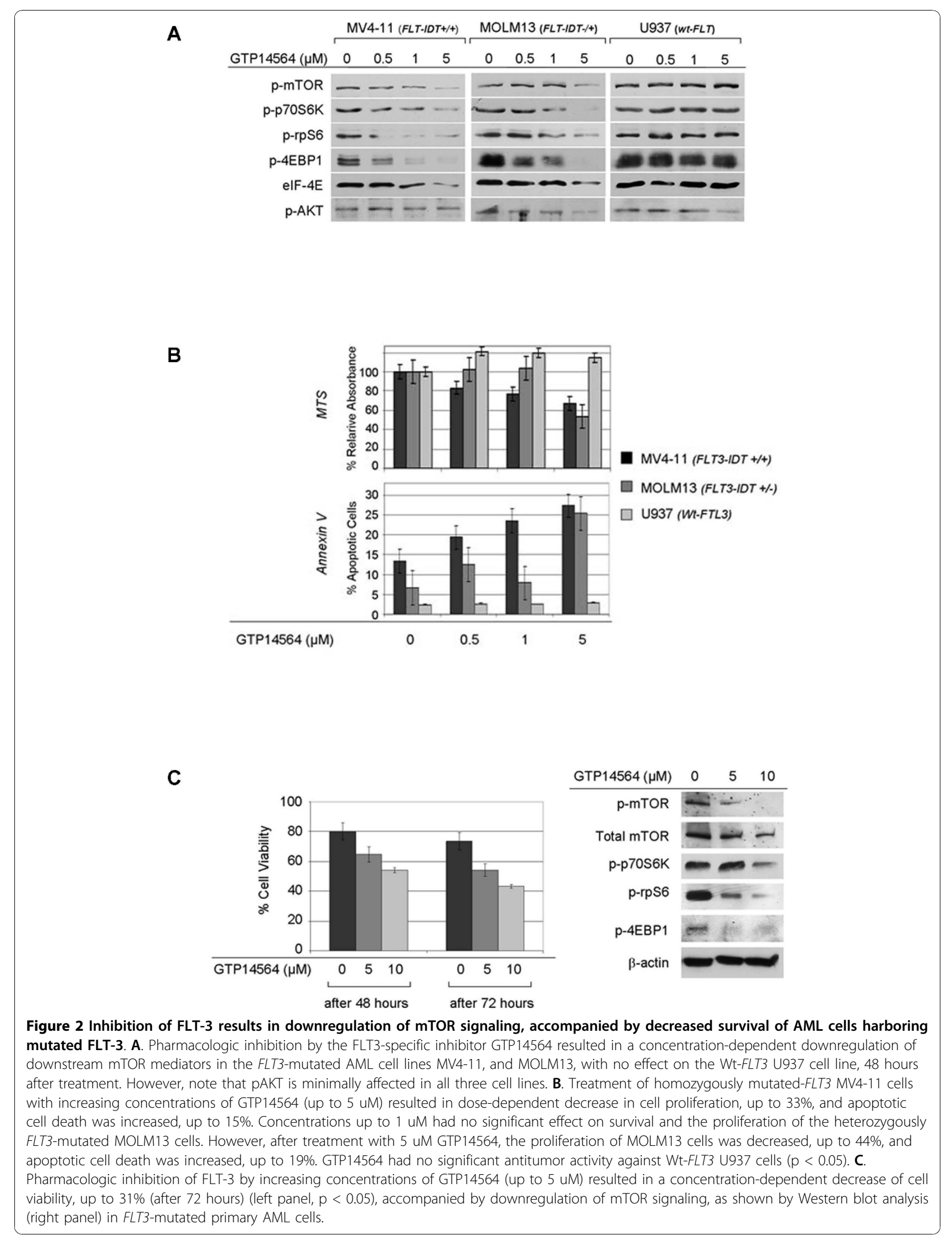


(Figure 2). These data provide evidence that mTOR signaling is an important downstream mediator of the oncogenic pathway activated by mutated FLT3 kinase (Figure 3), and that direct inhibition of mTOR may possibly augment the therapeutic potential of FLT3 inhibitors [16]. Our findings are in agreement with previous observations that rapamycin in combination with the FLT3 inhibitor, PKC-412, markedly inhibits cell proliferation in murine BaF-FLT3-ITD cells and AML samples bearing FLT3 gene dual mutations of ITD and point mutation types, and that rapamycin inhibits cell proliferation in murine 32D-FLT3-ITD cells [2,8,17].

\section{Conclusions}

In conclusion, our results taken together suggest that FLT3 mutations may lead to activation of mTOR signaling pathway and thus contribute to tumor cell survival and growth in AML (Figure 3). Therefore, selective inhibition of mTOR signaling pathway seems to be a promising therapeutic target for patients with FLT3mutated AML.

\section{Methods}

\section{Cells and Reagents}

Three human AML cell lines were used; MOLM-13 (heterozygous for FLT3-ITD), MV4-11 (homozygous for FLT3-ITD) [18], U937 (wild-type FLT3), and murine BaF3 cells transfected with wild-type (Wt) FLT3 (BaF3/ FLT3) or mutated FLT3 (FLT3/ITD and FLT3/D835G) were cultured under standard conditions as previously described [18]. The murine cell line BaF3 was kindly provided by Dr. M Andreeff (M.D. Anderson Cancer Center, Houston, TX). All four cell lines (MOLM-13, MV4-11, U937, BaF3) were tested negative for mycoplasma by

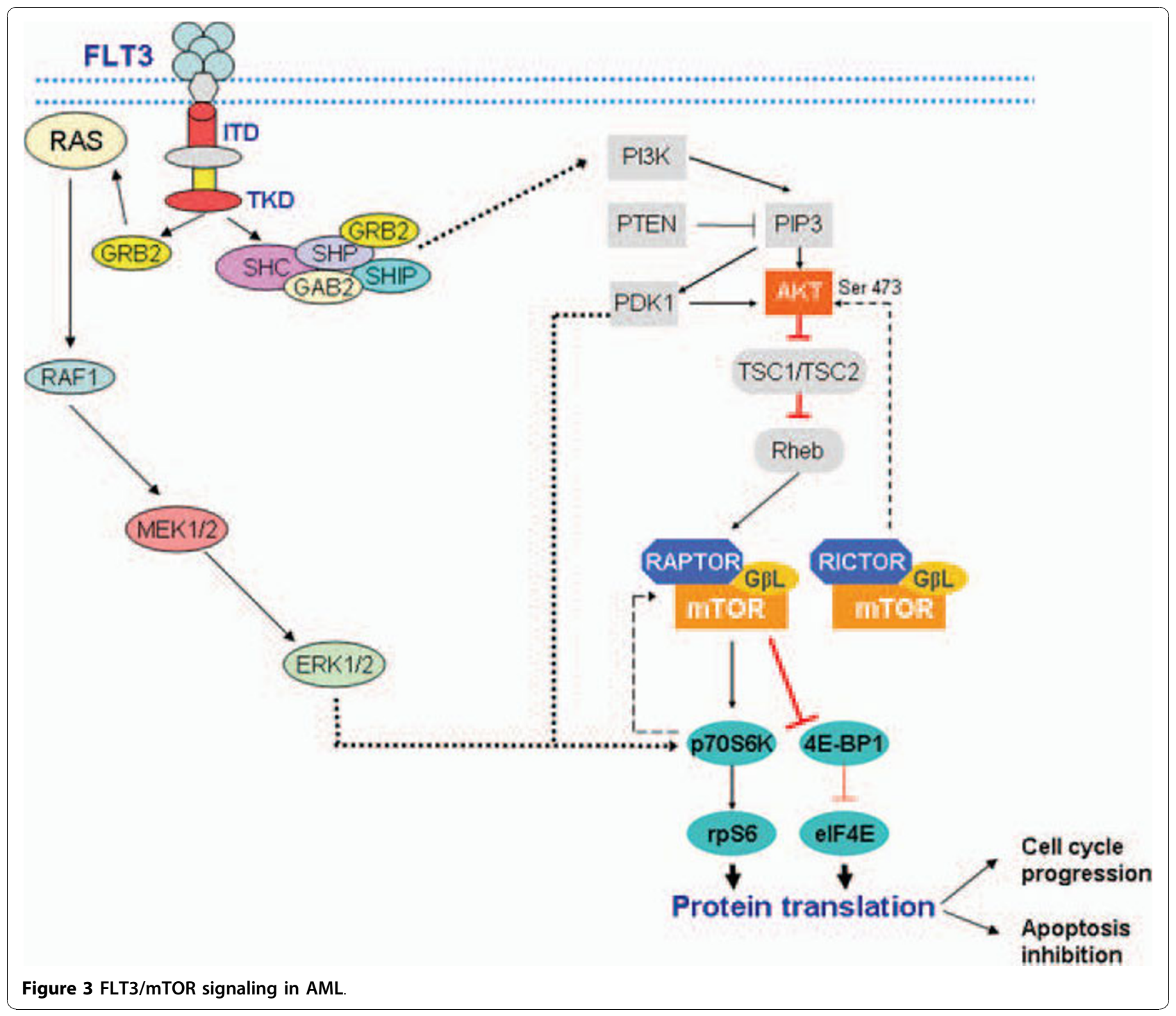


standard polymerase chain reaction (PCR) methods. Ficoll-purified (Sigma Chemical, St Louis, MO) peripheral blood mononuclear cells from 4 AML patients having $>60 \%$ blasts in blood and harboring FLT3-ITD mutations were also used for in vitro studies. The PI3K inhibitor LY294002, the mTOR inhibitor rapamycin and the FLT3 inhibitor GTP-14564 (all from Calbiochem. San Diego, CA) were applied at different concentrations and durations of time as indicated. The kinase inhibition experiments were performed at least three times with reproducible data.

\section{Immunohistochemistry}

Fifteen formalin-fixed, paraffin-embedded, AML bone marrow samples (9 harboring FLT3-ITD mutation, and 6 harboring dual, FLT3-ITD and point mutations) were used for the construction of a tissue microarray. Immunohistochemistry was performed using histologic sections of the tissue microarray or cell blocks, and antibodies specific for p-mTOR, p-p70S6K, p-rpS6, p-4E-BP1, and eIF4E (Cell Signaling Technology) as previously described [19]. AML cases were considered immunohistochemically positive for any of the proteins analyzed if at least $10 \%$ of tumor cells showed expression after examination of 10 representative high-power fields (magnification $\times 400$ ). The study was approved by The University of Texas M.D. Anderson Cancer Center institutional review board.

\section{Western Blot Analysis}

Western blot analysis was performed as previously described[19]. The antibodies used included ${ }^{\text {Ser473 }}$ pAKT (p-pAKT), total Akt, p-mTOR, p-p70S6K, p70S6K, p-rpS6, rpS6, p4E-BP1, 4E-BP1, eIF4E (Cell Signaling Technology, Beverly, MA), and $\beta$-actin (Sigma, St. Louis, MO).

\section{Silencing of mTOR by siRNA}

The sequences of $m T O R$ small interfering RNA (siRNA) (5'-GGAGUCUACUCGCUUCUAUTT-3' [sense]; 5'AUAGAAGCGAGUAGACUCCTC-3' [antisense] and a negative control siRNA were purchased from Ambion, Inc, (Austin, TX). Transient transfection was performed by electroporation using nucleofector system (Amaxa Biosystems, Gaithersburg, MD), according to the manufacturer's instructions. Gene silencing experiments were performed twice with reproducible results.

\section{Cell Death, Apoptosis and Proliferation Studies}

Cell viability was evaluated by trypan blue exclusion cell assay in triplicate. Annexin-V staining (BD Biosciences Pharmingen, San Diego, CA), detected by flow cytometry, was used to assess apoptosis, as previously described [20].
Proliferation of the cells was assessed by using a tetrazolium compound [3-(4,5-dimethylthiazol-2-yl)-5-(3-carboxymethoxyphenyl)-2-(4-sulfophenyl)-2H-tetrazolium, MTS] (Promega, Madison, WI, USA), as previously described [20]. All experiments were performed at least twice.

\section{Statistical analysis}

Data for cell viability, growth and apoptosis are shown in diagrams as mean +/- SD and their differences between different cell lines and concentrations were evaluated using Student $\mathrm{t}$-test. p-values $<0.05$ were considered statistically significant.

\section{Additional material}

Additional file 1: Inhibition of the AKT-mTOR signaling pathway results in apoptotic cell death of AML cells harboring mutated FLT3 (Figure S1). A. Pharmacologic inhibition of the AKT-mTOR signaling pathway by using $10 \mu \mathrm{g} / \mathrm{ml}$ LY249002 resulted in decreased cell viability and apoptotic cell death by $32 \%$ and $31 \%$ respectively in MOLM13 cells harboring heterozygously mutated FLT3 and by $48 \%$ and $75 \%$

respectively in MV4-11 cells harboring homozygously mutated FLT3, 48 hours after treatment $(p<0.05)$ (Figure S1A). B. Western blot analysis showed that LY249002-mediated cell death was accompanied by

downregulation of the inhibitor of the extrinsic apoptotic pathway FLIP and the inhibitor of the intrinsic apoptotic pathway BCL-XL in both MOLM13 and MV4-11 cells (Figure S1B).

Additional file 2: Combined treatment of AML cells with a FLT-3 inhibitor and a small dose of rapamycin results in enhanced cytotoxicity specifically in AML cells harboring mutated FLT-3 (Figure S2). Combination of $5 \mathrm{nM}$ GTP14564 with $1 \mathrm{nM}$ rapamycin resulted in enhanced apoptotic cell death, by $28 \%$ and $63 \%$, in MOLM13 and MV4-11 cells harboring heterozygously and homozygously mutated FLT3 respectively $(p<0.05)$ (Figure S2), as compared to $19 \%$ and 15\% induced by $5 \mathrm{nM}$ GTP14564 alone (Figure 2). No effect was observed in U937 cells harboring wt-FLT3.

Competing interests

The authors declare that they have no competing interests.

\section{Acknowledgements}

This study was funded by the NIH Joe Moakley Leukemia SPORE grant to The University of Texas M.D. Anderson Cancer Center. G.Z. Rassidakis is a recipient of a Leukemia SPORE Developmental Research Award.

\section{Authors' contributions}

WC designed and interpreted experiments and contributed to the writing of the manuscript, ED interpreted experiments and contributed to the writing of the manuscript, IG executed experiments, EJS contributed vital reagents and contributed to the writing of the manuscript, $J$, VL and ESD executed experiments, PP and EP contributed vital reagents and contributed to the writing of the manuscript, LJM interpreted experiments and contributed to the writing of the manuscript, and GZR designed and interpreted experiments and contributed to the writing of the manuscript. All authors have read and approved the final manuscript.

\section{Author details}

${ }^{1}$ Department of Hematopathology, The University of Texas M.D. Anderson Cancer Center, 1515 Holcombe Blvd., Houston, Texas 77030, USA. ${ }^{2}$ First Department of Pathology, National and Kapodistrian University of Athens, School of Medicine, 75 Mikras Asias str., Athens, 11527, Greece. ${ }^{3}$ Department 
of Hematology, National and Kapodistrian University of Athens, Laiko General Hospital, 17 Agiou Thoma str., Athens, 11527 Greece.

Received: 17 May 2010 Accepted: 10 November 2010

Published: 10 November 2010

\section{References}

1. Scheijen B, Griffin JD: Tyrosine kinase oncogenes in normal hematopoiesis and hematological disease. Oncogene 2002, 21:3314-3333.

2. Brandts $\mathrm{CH}$, Sargin B, Rode M, Biermann C, Lindtner B, Schwable J, Buerger H, Muller-Tidow C, Choudhary C, McMahon M, et al: Constitutive activation of Akt by Flt3 internal tandem duplications is necessary for increased survival, proliferation, and myeloid transformation. Cancer Res 2005, 65:9643-9650.

3. Arber DA, Brunning RD, Le Beau MM, Falini B, Vardiman J, Porwit A, Thiele J, Bloomfield CD: Acute myeloid leukemia with recurent genetic abnormalities. In World Health Organization Classification of Tumours of Haematopoietic and Lymphoid Tissues.. 4 edition. Edited by: Swerdlow SH, Campo E, Harris NL, Jaffe ES, Pileri SA, Stein H, Thiele J, Vardiman JW. Lyon, France: IARC Press; 2008:110-123, World Health Organization Classification of Tumours.

4. Guertin DA, Sabatini DM: Defining the role of mTOR in cancer. Cancer Cell 2007, 12:9-22.

5. Ma XM, Blenis J: Molecular mechanisms of mTOR-mediated translational control. Nat Rev Mol Cell Biol 2009, 10:307-318.

6. Sarbassov DD, Guertin DA, Ali SM, Sabatini DM: Phosphorylation and regulation of Akt/PKB by the rictor-mTOR complex. Science 2005, 307:1098-1101.

7. Recher C, Beyne-Rauzy O, Demur C, Chicanne G, Dos Santos C, Mas VM, Benzaquen D, Laurent G, Huguet F, Payrastre B: Antileukemic activity of rapamycin in acute myeloid leukemia. Blood 2005, 105:2527-2534.

8. Mohi MG, Boulton C, Gu TL, Sternberg DW, Neuberg D, Griffin JD, Gilliland DG, Neel BG: Combination of rapamycin and protein tyrosine kinase (PTK) inhibitors for the treatment of leukemias caused by oncogenic PTKs. Proc Natl Acad Sci USA 2004, 101:3130-3135.

9. Tibes R, Kornblau SM, Qiu Y, Mousses SM, Robbins C, Moses T, Carpten JD: $\mathrm{PI} 3 \mathrm{~K} / \mathrm{AKT}$ pathway activation in acute myeloid leukaemias is not associated with AKT1 pleckstrin homology domain mutation. $\mathrm{Br} J$ Haematol 2008, 140:344-347.

10. Zeng Z, Sarbassov dos D, Samudio IJ, Yee KW, Munsell MF, Ellen Jackson C, Giles FJ, Sabatini DM, Andreeff M, Konopleva M: Rapamycin derivatives reduce mTORC2 signaling and inhibit AKT activation in AML. Blood 2007, 109:3509-3512.

11. Tamburini J, Chapuis N, Bardet V, Park S, Sujobert P, Willems L, Ifrah N, Dreyfus F, Mayeux P, Lacombe C, Bouscary D: Mammalian target of rapamycin (mTOR) inhibition activates phosphatidylinositol 3-kinase/Akt by up-regulating insulin-like growth factor-1 receptor signaling in acute myeloid leukemia: rationale for therapeutic inhibition of both pathways. Blood 2008, 111:379-382.

12. Guertin DA, Sabatini DM: The pharmacology of mTOR inhibition. Sci Signal 2009, 2:pe24.

13. Vlahos CJ, Matter WF, Hui KY, Brown RF: A specific inhibitor of phosphatidylinositol 3-kinase, 2-(4-morpholinyl)-8-phenyl-4H-1benzopyran-4-one (LY294002). J Biol Chem 1994, 269:5241-5248.

14. Cai D, Wang Y, Ottmann OG, Barth PJ, Neubauer A, Burchert A: FLT3-ITD-, but not BCR/ABL-transformed cells require concurrent Akt/mTor blockage to undergo apoptosis after histone deacetylase inhibitor treatment. Blood 2006, 107:2094-2097.

15. Weisberg E, Banerji L, Wright RD, Barrett R, Ray A, Moreno D, Catley L, Jiang J, Hall-Meyers E, Sauveur-Michel M, et al: Potentiation of antileukemic therapies by the dual PI3K/PDK-1 inhibitor, BAG956: effects on BCR-ABL- and mutant FLT3-expressing cells. Blood 2008, 111:3723-3734.

16. Bullinger L, Rucker FG, Kurz S, Du J, Scholl C, Sander S, Corbacioglu A, Lottaz C, Krauter J, Frohling S, et al: Gene-expression profiling identifies distinct subclasses of core binding factor acute myeloid leukemia. Blood 2007, 110:1291-1300.

17. Bagrintseva K, Geisenhof S, Kern R, Eichenlaub S, Reindl C, Ellwart JW, Hiddemann W, Spiekermann K: FLT3-ITD-TKD dual mutants associated with AML confer resistance to FLT3 PTK inhibitors and cytotoxic agents by overexpression of Bcl-x(L). Blood 2005, 105:3679-3685.
18. Quentmeier H, Reinhardt J, Zaborski M, Drexler HG: FLT3 mutations in acute myeloid leukemia cell lines. Leukemia 2003, 17:120-124.

19. Vega F, Medeiros LJ, Leventaki V, Atwell C, Cho-Vega JH, Tian L, Claret FX, Rassidakis GZ: Activation of mammalian target of rapamycin signaling pathway contributes to tumor cell survival in anaplastic lymphoma kinase-positive anaplastic large cell lymphoma. Cancer Res 2006, 66:6589-6597.

20. Peponi E, Drakos E, Reyes G, Leventaki V, Rassidakis GZ, Medeiros L: Activation of mammalian target of rapamycin signaling promotes cell cycle progression and protects cells from apoptosis in mantle cell lymphoma. Am J Pathol 2006, 169:2171-2180.

doi:10.1186/1476-4598-9-292

Cite this article as: Chen et al:: mTOR signaling is activated by FLT3 kinase and promotes survival of FLT3-mutated acute myeloid leukemia cells. Molecular Cancer 2010 9:292.

\section{Submit your next manuscript to BioMed Central and take full advantage of:}

- Convenient online submission

- Thorough peer review

- No space constraints or color figure charges

- Immediate publication on acceptance

- Inclusion in PubMed, CAS, Scopus and Google Scholar

- Research which is freely available for redistribution

Submit your manuscript at www.biomedcentral com/submit
C Biomed Central 